\title{
Determination of Torque Distribution Ratio for Electric Bicycle with Independently Driven Front and Rear Wheels
}

\author{
Sho Sakaino* Senior Member, Hiroyuki Kawajiri* Non-member \\ Toshiaki Tsuji* Senior Member
}

(Manuscript received July 11, 2016, revised Nov. 17, 2016)

\begin{abstract}
Determining the torque distribution ratio for electric bicycles with independently driven front and rear wheels is important. Both wheels should be driven properly to ensure the safety of riders, and the slip ratio, which indicates the slip state, should be the same for both wheels. In this paper, a method to determine the torque distribution by making the slip ratios of both wheels equal is proposed. The validity of the proposed method is verified by simulations and experiments.
\end{abstract}

Keywords: electric bicycle, Kalman filter, torque distribution ratio

\section{Introduction}

Electric bicycles are eco-friendly vehicles because they do not emit carbon dioxide in working. These two-wheeled vehicles, however, are unstable compared with four-wheeled vehicles. Beznos et al. proposed gyroscopic stabilization control of two-wheeled vehicles ${ }^{(1)}$. Yamakita et al. reported automatic control of two-wheeled vehicles ${ }^{(2)}$. In addition, there are many research to stabilize two-wheeled vehicles $^{(3)-(6)}$. If two-wheeled vehicles can be made more stable, they will be more widely used. Friction force between tires and road surfaces reduces with idling of wheels. Therefore, idling of wheels is a factor that destabilizes two-wheeled vehicles. The idling condition is represented by slip ratios. Hence, slip ratio control systems have been developed ${ }^{(8)(9)}$. Another way to avoid slip is increment of driving wheels. By increasing driving wheels, the driving force per wheel can be decreased. Owing to this, the driving force of a wheel becomes smaller than the maximum frictional force between tires and road surfaces. Therefore, we developed an electric bicycle, in which the front and rear wheels can be independently driven.

The torque distribution ratio between the front and rear wheels must be determined when the proposed two-wheeled vehicle is driven. The torque distribution ratio is related to each wheel's slip ratio. Therefore, the slip ratio of each wheel should be considered while determining the torque distribution ratio. A wheel's excessive idling is avoided by equalizing the slip ratio of each wheel. In four-wheeled vehicles, control systems exist for suppressing the difference between the front and rear wheels' slip ratios ${ }^{(10)}$. Such systems are realized by determining the torque distribution ratio using the reaction force distribution ratio between the front and rear wheels. The reaction force distribution ratio depends on the

\footnotetext{
* Graduate School of Science and Engineering, Saitama University

255, Shimo-okubo, Sakura-ku, Saitama 338-8570, Japan
}

gravity point's position and vehicle acceleration. However, knowing the center of gravity point is difficult, because postures of riders and positions of baggage are difficult to know in advance. In addition, almost no electric bicycles have acceleration sensors. Obtaining correct data of a vehicle's acceleration by using low-resolution encoders is difficult.

In this paper, a torque distribution ratio control method is proposed where slip ratios are equalized without knowing the reaction force distribution and vehicle's acceleration. In the proposed method, a model of the slip ratio difference and the torque distribution is described. The model's parameters are identified by the Kalman filter. Owing to this, the appropriate torque distribution ratio can be determined even without knowing the reaction force distribution and vehicle's acceleration. The slip ratio of each wheel is equalized by using the appropriate torque distribution ratio estimated by using the proposed method. In addition, Fujimoto et al. recently reported that driving range of electric vehicles can be extended with adequate torque distributions ${ }^{(11)}$. Therefore, the proposed method can also be contributed for the driving range extension. The validity of the proposed method is confirmed by simulations and experiments.

The organization of the present paper is as follows. A model of the two-wheeled electric bicycle is shown in section 2. A controller for the two-wheeled electric bicycle is described in section 3 . The performance of the proposed method is discussed in section 4. Simulation results are presented in section 5, and experimental results are presented in section 6 . Section 6 concludes the paper.

\section{Modeling}

In this section, a model that relates the difference of each wheel's slip ratio and the torque distribution ratio is obtained from the motion equations of two-wheeled vehicles. Figure 1 shows a vehicle model. All the parameters of the vehicle are listed in Table 1, and the variables are defined in Table 2. Note that the parameters related to COG positions, 


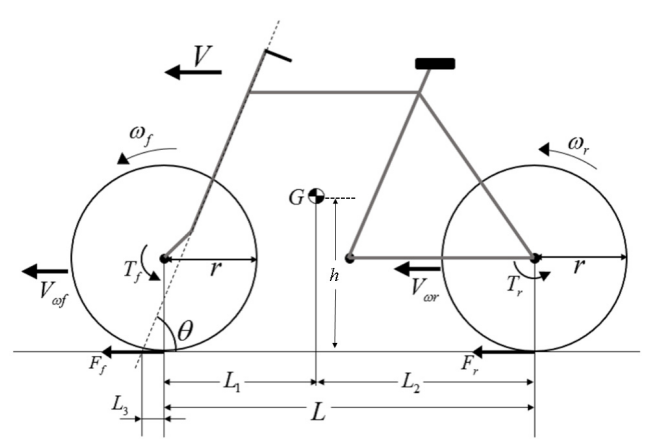

Fig. 1. Vehicle model

Table 1. Parameter of vehicle

\begin{tabular}{|c|c|c|}
\hline$M$ & Mass [kg] & 120.0 \\
\hline$L$ & Wheelbase $[\mathrm{m}]$ & 1.05 \\
\hline$L_{1}$ & Distance from Center of Gravity to Front Wheel [m] & 0.4 \\
\hline$L_{2}$ & Distance from Center of Gravity to Rear Wheel [m] & 0.6 \\
\hline$L_{3}$ & Trail $[\mathrm{m}]$ & 0.22 \\
\hline$h$ & Center of Gravity to Road Surface [m] & 1.0 \\
\hline$\theta$ & Caster Angle [deg] & 61.0 \\
\hline$J_{f}$ & Inertia of Front Wheel $\left[\mathrm{kgm}^{2}\right]$ & 0.12 \\
\hline$J_{r}$ & Inertia of Rear Wheel $\left[\mathrm{kgm}^{2}\right]$ & 0.12 \\
\hline$r$ & Wheel Radius [m] & 0.35 \\
\hline & Angle measurement resolution of wheel [mrad] & 1.571 \\
\hline
\end{tabular}

Table 2. Definition of variables

\begin{tabular}{|c||c|}
\hline$\omega_{f}$ & Angular Velocity of Front Wheel [rad/sec] \\
$\omega_{r}$ & Angular Velocity of Rear Wheel [rad/sec] \\
$T_{\text {sum }}$ & Total Torque [Nm] \\
$T_{f}$ & Motor Torque (front) [Nm] \\
$T_{r}$ & Motor Torque (rear) [Nm] \\
$K$ & Torque distribution ratio \\
$F_{f}$ & Driving Force (front) [N] \\
$F_{r}$ & Driving Force (rear) [N] \\
$N_{f}$ & Reaction Force (front) [N] \\
$N_{r}$ & Reaction Force (rear) [N] \\
$D_{f}$ & Gradient of Friction Coefficient (front) \\
$D_{r}$ & Gradient of Friction Coefficient (rear) \\
$\mu_{f}$ & Friction Coefficient (front) \\
$\mu_{r}$ & Friction Coefficient (rear) \\
$\lambda_{f}$ & Slip Ratio (front) \\
$\lambda_{r}$ & Slip Ratio (rear) \\
$V$ & Vehicle Velocity [m/s] \\
$F_{d}$ & Running Resistance [N] \\
\hline
\end{tabular}

$L_{1}, L_{2}$, and $h$, are used only in a dynamics simulator while the proposed controller does not require the parameters. The front and rear wheels' motion equations are given in (1) and (2). Subscripts $f$ and $r$ represent the front wheel and the rear wheel, respectively.

$$
\begin{aligned}
& J_{f} \dot{\omega}_{f}=T_{f}-r F_{f} \\
& J_{r} \dot{\omega}_{r}=T_{r}-r F_{r} .
\end{aligned}
$$

In this paper, inertial forces of wheels are assumed to be negligibly small, and Eqs. (1) and (2) are simplified as (3) and (4).

$$
\begin{aligned}
& T_{f} \simeq r F_{f} \\
& T_{r} \simeq r F_{r}
\end{aligned}
$$

Motor torques of the front and rear wheels are decided by (5) and (6). The total torque $T_{\text {sum }}$ is distributed by torque distribution ratio $K$.

$$
T_{f}=K T_{\text {sum }}
$$

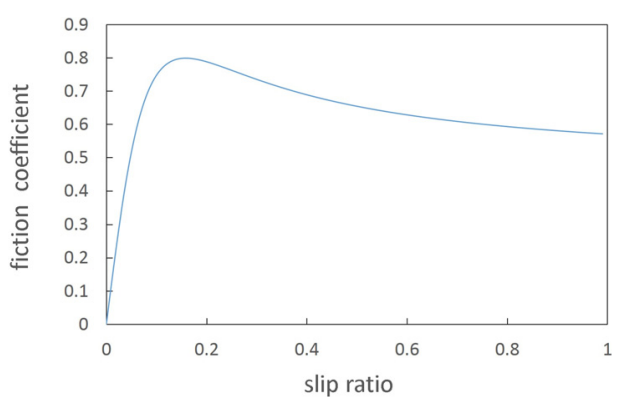

Fig. 2. Characteristic of friction coefficient

$$
T_{r}=(1-K) T_{\text {sum }}
$$

Reaction forces of front and rear wheels are shown in (7) and (8), and are determined by using the expressions for force and moments of balance.

$$
\begin{aligned}
& N_{f}=\frac{L_{2}}{L} M g-\frac{h}{L} M \dot{V} \\
& N_{r}=\frac{L_{1}}{L} M g+\frac{h}{L} M \dot{V}
\end{aligned}
$$

Dynamic friction forces are generated between tires and road surfaces during driving. The driving forces are determined using (9) and (10).

$$
\begin{aligned}
& F_{f}=\mu_{f} N_{f} \\
& F_{r}=\mu_{r} N_{r} .
\end{aligned}
$$

Front and rear wheels' slip ratios in acceleration are defined in (11) and (12). The slip ratio represents idling conditions of the wheels.

$$
\begin{aligned}
& \lambda_{f}=\frac{r \omega_{f}-V}{r \omega_{f}} . \\
& \lambda_{r}=\frac{r \omega_{r}-V}{r \omega_{r}} .
\end{aligned}
$$

Relationships between the slip ratios and the friction coefficients are non-linear. An example of the relationship between them is shown in Fig. 2. When the slip ratio is small, the relationships can be considered to be approximately linear. This assumption is also used in other research ${ }^{(12)}$. Owing to this, relationships between slip ratios and the friction coefficients are linearized as shown in (13) and (14).

$$
\begin{aligned}
& \mu_{f}=D_{f} \lambda_{f} \\
& \mu_{r}=D_{r} \lambda_{r} .
\end{aligned}
$$

The vehicle's motion equation is written as (15).

$$
M \dot{V}=F_{f}+F_{r}+F_{d}
$$

Equation (16) is obtained from (3), (4), (5), (6), and (15).

$$
\dot{V}=\frac{T_{\text {sum }}}{r M}+\frac{F_{d}}{M}
$$

Equations (17) and (18) are obtained from (3), (9), and (13); and (4), (10), and (14), respectively.

$$
\begin{aligned}
& \lambda_{f}=\frac{T_{f}}{r D_{f} N_{f}} \\
& \lambda_{r}=\frac{T_{r}}{r D_{r} N_{r}}
\end{aligned}
$$


Normal forces are transformed into (19) and (20) by using (7), and (16), and (8), and (16), respectively.

$$
\begin{aligned}
& N_{f}=\frac{L_{2}}{L} M g-\frac{h}{L}\left(\frac{T_{\text {sum }}}{r}+F_{d}\right) \\
& N_{r}=\frac{L_{1}}{L} M g+\frac{h}{L}\left(\frac{T_{\text {sum }}}{r}+F_{d}\right)
\end{aligned}
$$

The difference of the slip ratios is given in (21) obtained from (5), (6), (17), and (18).

$$
\lambda_{f}-\lambda_{r}=\frac{K T_{\text {sum }}}{r D_{f} N_{f}}-\frac{(1-K) T_{\text {sum }}}{r D_{r} N_{r}} .
$$

Finally, the difference of the slip ratios is parameterized in (22) by using (19), (20) and (21)

$$
\lambda_{f}-\lambda_{r}=\frac{K T_{\text {sum }}}{A-B T_{\text {sum }}}-\frac{(1-K) T_{\text {sum }}}{C+D T_{\text {sum }}} .
$$

where $A, B, C$, and $D$ are defined by (23)-(26).

$$
\begin{aligned}
A & =\frac{D_{f} r}{L}\left(L_{2} M g-F_{d} h\right) . \\
B & =\frac{D_{f} h}{L} \ldots \ldots \ldots \ldots \ldots \\
C & =\frac{D_{r} r}{L}\left(L_{1} M g+F_{d} h\right) . \\
D & =\frac{D_{r} h}{L} \ldots \ldots \ldots \ldots
\end{aligned}
$$

Equation (22) is a nonlinear equation. In this paper, (22) is linearized by the Taylor expansion for $T_{\text {sum }}$ as follows: [see Appendix]

$$
\lambda_{f}-\lambda_{r} \risingdotseq T_{\text {sum }}\left(\alpha K+\beta T_{\text {sum }}+\gamma\right) .
$$

In this study, $\alpha, \beta$, and $\gamma$ are treated as unknown parameters because mass, center of gravity, gradient of friction coefficient, and running resistance are variables, and actual values are unknown. Equation (28) is obtained from (11) and (12).

$$
\lambda_{f}-\lambda_{r}=\frac{V \omega_{f}-V \omega_{r}}{r \omega_{f} \omega_{r}}
$$

When $\frac{V}{r \omega_{f}} \simeq 1$ is assumed, the difference of each wheel slip ratio is approximately calculated by (29).

$$
\lambda_{f}-\lambda_{r} \simeq \frac{\omega_{f}-\omega_{r}}{\max \left(\omega_{r}, v\right)}
$$

When Eq. (29) is used, the signal-to-noise ratio is very poor in the case of $w_{r}$ being very small. Hence, $v$ is introduced to avoid zero division. $v$ was set as 0.01 . In this study, the difference of each wheel slip ratio data is obtained from (29) without knowing vehicle velocity. Roll angle and steering angle are not considered for simplifying the discussion but the proposed method can be applied to a system with variation in roll and steering angles with some modifications.

\section{Kalman Filter}

In this section, a method for identifying $\alpha, \beta$, and $\gamma$ by using the Kalman filter is explained. The Kalman filter is promising technique to identify system parameters ${ }^{(13)-(15)}$. The linear discrete-time state-space equations of the difference of each wheel's slip ratio are given in (30)-(36). $v(k)$ and $n(k)$ are respectively a system noise vector and an observation noise. $k$ represents discrete-time.

$$
\begin{aligned}
& \boldsymbol{x}(k+1)=\boldsymbol{A} \boldsymbol{x}(k)+\boldsymbol{B} \boldsymbol{v}(k) \\
& y(k)=\boldsymbol{C} \boldsymbol{x}(k)+n(k) \ldots \ldots \ldots \\
& \boldsymbol{A}=\left(\begin{array}{lll}
1 & 0 & 0 \\
0 & 1 & 0 \\
0 & 0 & 1
\end{array}\right) \ldots \ldots \ldots \ldots \\
& \boldsymbol{B}=\left(\begin{array}{lll}
1 & 0 & 0 \\
0 & 1 & 0 \\
0 & 0 & 1
\end{array}\right) \ldots \ldots \ldots \ldots \\
& \boldsymbol{C}=\left(\begin{array}{ll}
K T_{\text {sum }} & T_{\text {sum }}^{2} T_{\text {sum }}
\end{array}\right) \\
& \boldsymbol{x}=\left(\begin{array}{l}
\alpha \\
\beta \\
\gamma
\end{array}\right) \ldots \ldots \ldots \ldots \ldots \\
& y=\lambda_{f}-\lambda_{r} \ldots \ldots \ldots \ldots .
\end{aligned}
$$

The Kalman filter consists of two steps. The first step is the prediction step. In the prediction step, a pre-state estimate vector $\hat{\boldsymbol{x}}^{-}(k)$ and a pre-error covariance matrix $\hat{\boldsymbol{P}}^{-}(k)$ are respectively calculated by (37) and (38).

$$
\begin{aligned}
& \hat{\boldsymbol{x}}^{-}(k)=\boldsymbol{A} \hat{\boldsymbol{x}}(k-1) \ldots \ldots \ldots \ldots \\
& \boldsymbol{P}^{-}(k)=\boldsymbol{A} \boldsymbol{P}(k-1) \boldsymbol{A}^{T}+\boldsymbol{B} \boldsymbol{Q} \boldsymbol{B}^{T}
\end{aligned}
$$

$\boldsymbol{P}$ and $\boldsymbol{Q}$ are respectively a posteriori error covariance matrix and covariance matrix of $\boldsymbol{v}(\mathrm{k})$. The second step of the Kalman filter is the filtering step. The Kalman gain vector $\boldsymbol{G}(k)$, the state estimate vector $\hat{\boldsymbol{x}}(k)$, and the posteriori error covariance matrix $\boldsymbol{P}(k)$ are calculated using (39)-(41) in the filtering step.

$$
\begin{aligned}
& \boldsymbol{G}(k)=\boldsymbol{P}^{-}(k) \boldsymbol{C}^{T}\left(\boldsymbol{C} \boldsymbol{P}^{-}(k) \boldsymbol{C}^{T}+R\right)^{-1} \\
& \hat{\boldsymbol{x}}(k)=\hat{\boldsymbol{x}}^{-}(k)+\boldsymbol{G}(k)\left(y(k)-\boldsymbol{C} \hat{\boldsymbol{x}}^{-}(k)\right) \\
& \boldsymbol{P}(k)=(\boldsymbol{I}-\boldsymbol{G}(k) \boldsymbol{C}) \boldsymbol{P}^{-}(k) \ldots \ldots \ldots \ldots
\end{aligned}
$$

$R$ represents the variance of the observation noise in (39). The state estimation vector is updated in real-time by updating (37)-(41). Equation (42) is obtained from (27).

$$
K=\frac{\lambda_{f}-\lambda_{r}}{\alpha T_{\text {sum }}}-\frac{\beta T_{\text {sum }}+\gamma}{\alpha} .
$$

In order to satisfy $\lambda_{f}-\lambda_{r}=0, K$ should be determined by (43).

$$
K=-\frac{\beta T_{\text {sum }}+\gamma}{\alpha}
$$

A block diagram of the proposed controller is shown in Fig. 3.

\section{Discussion}

The discussion about the performance of the proposed method is given here. The proposed method was compared with a feedback control system. The feedback control system was a speed difference control system used for suppressing the difference between the front and rear wheels' slip ratios ${ }^{(16)}$ based on modal decomposition ${ }^{(17)}$.

4.1 Feedback Control System The conventional 


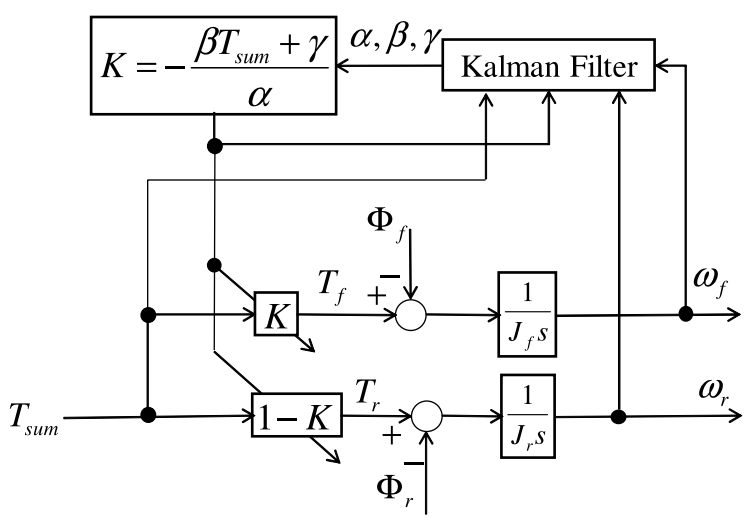

Fig. 3. Block diagram of proposed controller

controller is explained here. Disturbance torques of each wheel are described as $\Phi_{f}$ and $\Phi_{r}$, which are defined as (44) and (45). The disturbance torques were estimated by a disturbance observer ${ }^{(18)}$.

$$
\begin{aligned}
& \Phi_{f}=r F_{f} \\
& \Phi_{r}=r F_{r} .
\end{aligned}
$$

Equation (46) is obtained by (1), (2), (44), and (45).

$$
\dot{\omega}_{f}-\dot{\omega}_{r}=\left(\frac{T_{f}}{J_{f}}-\frac{T_{r}}{J_{r}}\right)+\left(\frac{\Phi_{r}}{J_{r}}-\frac{\Phi_{f}}{J_{f}}\right) .
$$

Variables $\dot{e}, \delta$, and $\Phi$ are defined by (47)-(49)

$$
\begin{aligned}
& e=\omega_{f}-\omega_{r} \cdots \\
& \delta=\left(\frac{T_{f}}{J_{f}}-\frac{T_{r}}{J_{r}}\right) \\
& \Phi=\left(\frac{\Phi_{f}}{J_{f}}-\frac{\Phi_{r}}{J_{r}}\right)
\end{aligned}
$$

Then (46) is written as (50).

$$
\dot{e}=\delta-\Phi
$$

$\delta$ is calculated by (51).

$$
\delta=-K_{p} e+\hat{\Phi}
$$

$K_{p}$ represents proportional gain. Here, $\hat{\Phi}$ is an estimate value of $\Phi$, which is calculated by disturbance observers of each wheel. $T_{f}$ and $T_{r}$ are determined by using (52) and (53), which are obtained from (5), (6), (48), and (51).

$$
\begin{aligned}
& T_{f}=\frac{J_{f} T_{\text {sum }}-J_{f} J_{r}\left(K_{p} e-\hat{\Phi}\right)}{J_{f}+J_{r}} \\
& T_{r}=\frac{J_{r} T_{\text {sum }}+J_{f} J_{r}\left(K_{p} e-\hat{\Phi}\right)}{J_{f}+J_{r}} .
\end{aligned}
$$

A block diagram of the conventional controller is shown in Fig. 4.

Equations (50) and (51) yield the following equation.

$$
\dot{e}(t)+K_{p} e(t)=-\Phi(t)+\hat{\Phi}(t)
$$

Equation (29) and $e_{\lambda}=\lambda_{f}-\lambda_{r}$ yield

$$
e_{\lambda}(t)=\frac{e(t)}{\omega_{r}}
$$

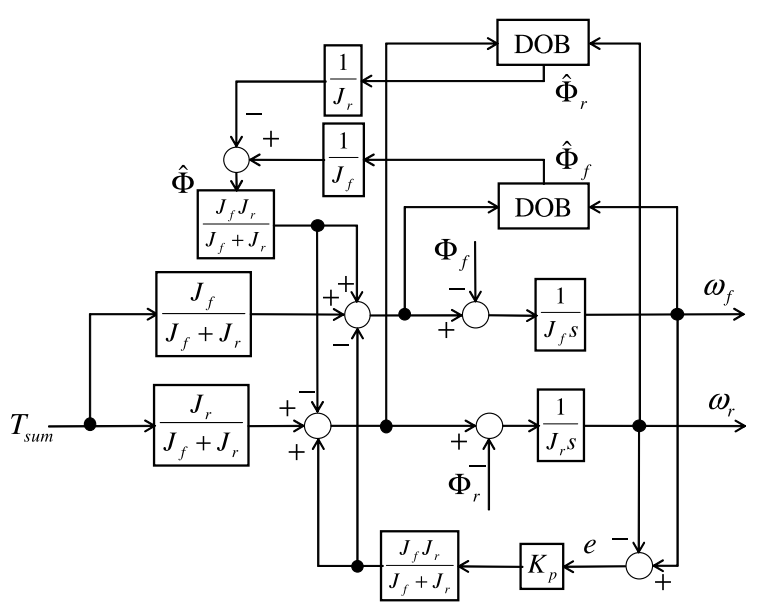

Fig. 4. Block diagram of conventional controller

with sufficiently large $\omega_{r}$. Then, (54) is transformed as follows:

$$
\begin{aligned}
& \omega_{r} \dot{e_{\lambda}}(t)+\left(K_{p} \omega_{r}+\dot{\omega}_{r}\right) e_{\lambda}(t)=-\Phi(t)+\hat{\Phi}(t) \\
& \dot{e_{\lambda}}(t)+\left(K_{p}+\frac{\dot{\omega}_{r}}{\omega_{r}}\right) e_{\lambda}(t)=\frac{1}{\omega_{r}}(-\Phi(t)+\hat{\Phi}(t)) \cdots
\end{aligned}
$$

Then, its steady state value is obtained by the final value theorem.

$$
\begin{aligned}
& E_{\lambda}(s)=\frac{1}{s+K_{p}+\frac{\dot{\omega}_{r}}{\omega_{r}}} \frac{1}{\omega_{r}}(-\Phi(s)+\hat{\Phi}(s)) \\
& \lim _{t \rightarrow \infty} e_{\lambda}(t)=\lim _{s \rightarrow 0} \frac{s}{s+K_{p}+\frac{\dot{\omega}_{r}}{\omega_{r}}} \frac{1}{\omega_{r}}(-\Phi(s)+\hat{\Phi}(s))
\end{aligned}
$$

Therefore, when estimation errors of disturbance torque $\frac{1}{\omega_{r}}(\Phi-\hat{\Phi})$ include $s$ or higher order denominator polynomials, steady state errors occur in the conventional method. Note that disturbance observers can only estimate step disturbances. As described in section 2, $\Phi_{f}$ and $\Phi_{r}$ are not step disturbances, e.g. friction coefficients $\mu_{f}$ and $\mu_{r}$ dependent on velocity, resulting in estimation errors and steady state errors. As a result, to eliminate steady state errors by feedback controllers, high feedback gains with high resolution sensors are essential.

4.2 Proposed Control System In the proposed method, slip ratio difference is not intended to converge to zero but the parameters related to slip ratio difference are determined by using the Kalman filter. As it can been found in (23) and (25), perturbation of running resistance $F_{d}$, which is a disturbance term in vehicle dynamics dimension, changes $A$ and $B$, and also $\alpha, \beta$, and $\gamma$. In the derivation of the Kalman filter, parameter perturbation is considered by the system noise vector $\boldsymbol{v}(k)$. However, the actual covariance of the system noise vector is unknown and it may deteriorate robustness of the controller. Then, the effect of the modeling errors of the system noise is discussed.

In steady state, $\boldsymbol{P}(k)=\boldsymbol{P}(k-1)=\boldsymbol{P}^{-}(k)=\boldsymbol{P}^{*}$ is established. Therefore, Eqs. (32), (33), (38), and (41), yield the following relation.

$$
\begin{aligned}
& \boldsymbol{P}^{*}=(\boldsymbol{I}-\boldsymbol{G}(k) \boldsymbol{C}) \boldsymbol{P}^{*}+\boldsymbol{Q} \\
& \boldsymbol{G}(k) \boldsymbol{C} \boldsymbol{P}^{*}=\boldsymbol{Q} \ldots \ldots \ldots
\end{aligned}
$$

This equation clearly shows that $\boldsymbol{G}(k)$ is a constant matrix $\boldsymbol{G}^{*}$ 
called as the steady state Kalman gain. By substituting $\boldsymbol{G}^{*}$ into (31), (32), (37), and (40), the dynamics in steady state is obtained.

$$
\begin{aligned}
& \hat{\boldsymbol{x}}(k)=\hat{\boldsymbol{x}}(k-1)+\boldsymbol{G}^{*}(\boldsymbol{C} \boldsymbol{x}(k)+n(k)-\boldsymbol{C} \hat{\boldsymbol{x}}(k-1)) \\
& \hat{\boldsymbol{x}}(k)=\left(z \boldsymbol{I}-\boldsymbol{I}+\boldsymbol{G}^{*} \boldsymbol{C}\right)^{-1}\left(z \boldsymbol{G}^{*} \boldsymbol{C} \boldsymbol{x}(k)+z \boldsymbol{G}^{*} n(k)\right) \cdots
\end{aligned}
$$

The above equation proofs that the steady state of the estimate $\hat{\boldsymbol{x}}(k)$ has no relation with the system noise $\boldsymbol{v}(k)$. Even with poor sensors, modeling errors gradually decrease in the proposed method thanks to time series data.

If low resolution sensors are equipped with the vehicle, determination of the parameters takes too much time. However, once adequate parameters are determined, slip ratio difference is immediately canceled. In other words, there is no phase delay of poles of controllers because the proposed method is a kind of adaptive feedforward controller. The proposed method makes up for the shortcoming of low resolution sensors by using time series data with the Kalman filter.

\section{Simulation}

In this section, the validity of the proposed method is confirmed by simulations. The relationship between the slip ratios and the friction coefficients was simulated by the Magic Formula $^{(19)}$. The Magic Formula's parameters were set as those corresponding to a dry road surface. The simulation conditions are given in Table 3 . In this paper, $t$ represents time. In case of $t<10, T_{\text {sum }}$ was determined by using (60) in the simulations.

$$
T_{\text {sum }}(t)=20+15 \sum_{i=0}^{2} \sin \left(3.14 \times 2^{i-2} t\right) \cdots \cdots \cdots
$$

In case of $t>10, T_{\text {sum }}$ was determined by using (61) in the simulations.

$$
T_{\text {sum }}(t)=1.75+15 \sum_{i=0}^{2} \sin \left(3.14 \times 2^{i-2} t\right) \ldots \ldots \ldots
$$

5.1 Condition of Kalman Filter The update of the Kalman Filter was started after $10 \mathrm{~s}$ from the beginning of the simulation. The conventional feedback controller was performed from the beginning of the simulation to $20 \mathrm{~s}$. Then, the controller was switched to the proposed control method shown in (43) after $20 \mathrm{~s}$.

5.2 Simulation Result The parameters used in the simulation and experiment are given in Table 4. Because the angle measuring resolution of wheels was not high enough, $1.571 \mathrm{mrad}$, the proportional gain and the cut-off frequency of the disturbance observer cannot be high. Then, the proportional gain and the cut-off were tuned to minimize tracking errors by trial and error. $R$ was determined to be greater than variance of sensing noises. In the simulations and experiments, because $\alpha, \beta$, and $\gamma$ hardly varied, $\boldsymbol{Q}$ was set as a zero matrix.

Time variation of the vehicle speed in the proposed method is shown in Fig. 5. A slip ratio difference in the proposed method is shown in Fig. 6, and that in the feedback controller is shown in Fig. 7, respectively. The difference of slip ratios in the proposed method was obviously smaller than that in the feedback controller.

Equations (19) and (20) show that when the total torque is
Table 3. Simulation condition

\begin{tabular}{|c|c|c|}
\hline$K_{p}$ & Proportional Gain & 1.2 \\
\hline$g_{d}$ & Cut-off Frequency of Disturbance Observer [rad/s] & 30 \\
\hline$T_{c}$ & Control Period [ms] & 1 \\
\hline \multirow[t]{2}{*}{$T_{K}$} & Update Cycle of Kalman Filter [ms] & 200 \\
\hline & & $\left(\begin{array}{lll}10 & 0 & 0\end{array}\right.$ \\
\hline \multirow[t]{3}{*}{$\boldsymbol{P}(0)$} & Initial Error Covariance Matrix & $\begin{array}{lll}0 & 10 & 0\end{array}$ \\
\hline & & $\begin{array}{lll}0 & 0 & 10\end{array}$ \\
\hline & & $\left(\begin{array}{lll}0 & 0 & 0\end{array}\right)$ \\
\hline \multirow[t]{2}{*}{$Q$} & System Noise Covariance Matrix & $\begin{array}{lll}0 & 0 & 0\end{array}$ \\
\hline & & $\left(\begin{array}{lll}0 & 0 & 0\end{array}\right.$ \\
\hline$R$ & Observation Noise Covariance Matrix & 0.1 \\
\hline$\hat{\boldsymbol{x}}(0)$ & Initial Estimate Vector & $\left(\begin{array}{lll}0 & 0 & 0\end{array}\right)^{T}$ \\
\hline
\end{tabular}

\begin{tabular}{|c||c|c|}
\hline$F_{d}$ & Running resistance $[\mathrm{N}]$ & 5 \\
$T_{p}$ & Physical simulation period [ns] & 100 \\
\hline
\end{tabular}

Table 4. Control parameter of simulation and experiment

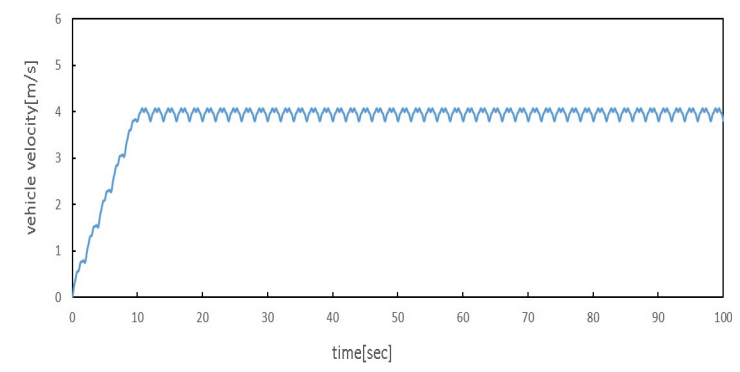

Fig. 5. Vehicle speed in simulation

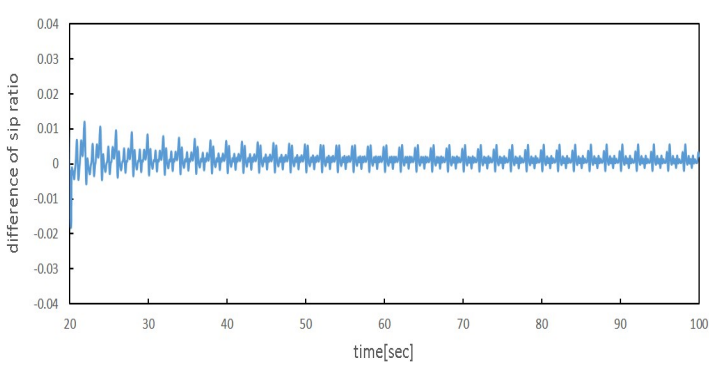

Fig. 6. Slip ratio difference in proposed method (simulation)

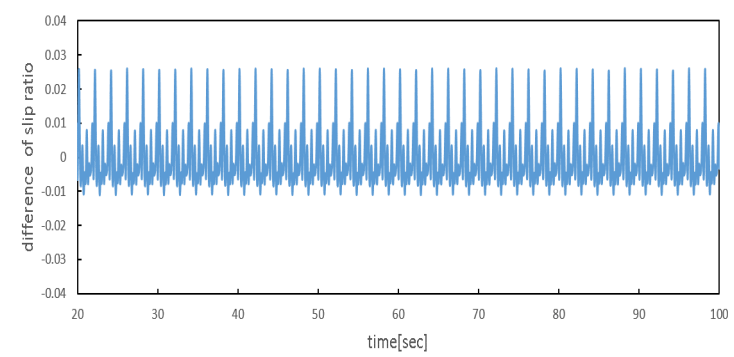

Fig. 7. Slip ratio difference in conventional controller (simulation)

large, the reaction force of the front wheel is reduced and the reaction force of the rear wheel is increased. Therefore, when total torque is large, torque distribution ratio should be small because a wheel with small reaction force should not output a large torque. Figure 8 shows the relation between the torque distribution ratio and the total torque in the proposed method. Figure 8 implies that the torque distribution ratio was appropriately adjusted by the proposed method according to the reaction force. We can summarize that the validity 


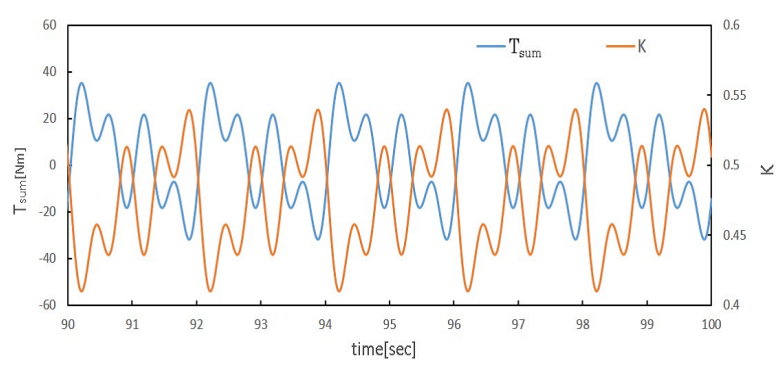

Fig. 8. Relation between total torque and torque distribution ratio of proposed method (simulation)

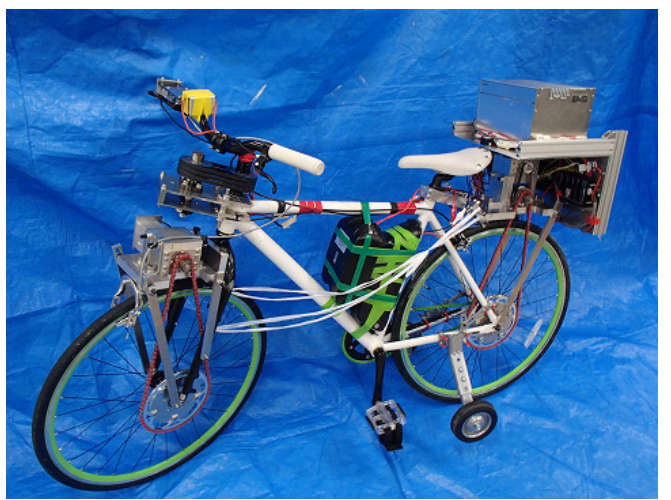

Fig. 9. Experimental vehicle

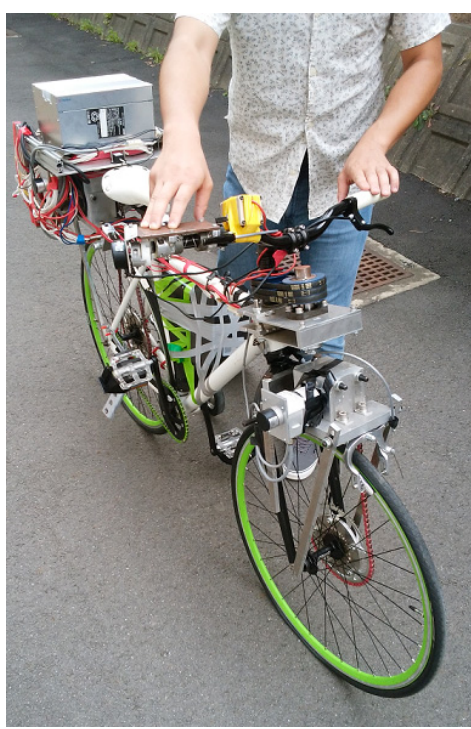

Fig. 10. Situation of experiment

of the proposed method was confirmed by Fig. 6, Fig. 7, and Fig. 8.

\section{Experimental Result}

In this section, experimental results are presented. The experimental system is shown in Fig. 9. The same setting parameters were used in the simulation. In the experiment of the proposed method, the vehicle was supported by an experimenter to prevent it from falling down. The experimenter supported the handlebars from the lateral direction so as not to affect any forces towards the vehicle's running direction, and ran with the bike at the same speed when the vehicle moved as shown in Fig. 10. We could not prepare a long straight road because of limitation of the experimental space.

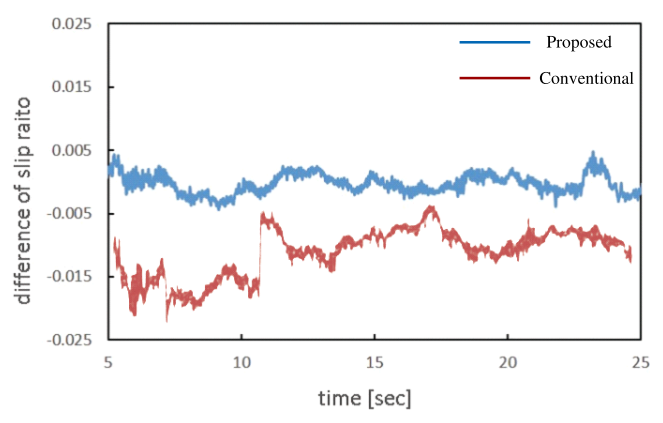

Fig. 11. Slip ratio difference (experiment)

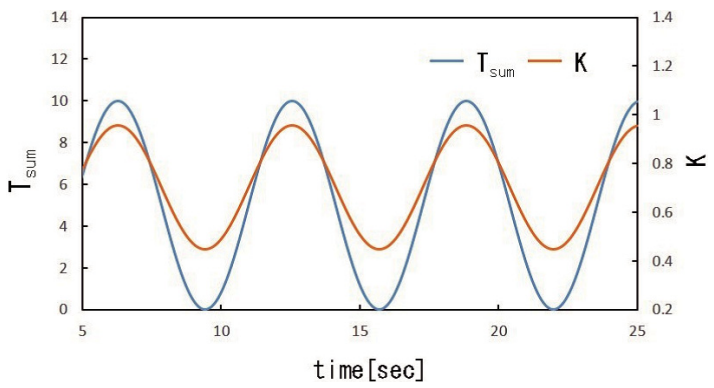

Fig. 12. Relation between total torque and torque distribution ratio of proposed method (experiment)

Therefore, the duration of the experiments was shorter than that of the simulations. $T_{\text {sum }}$ was determined by using (62) in these experiments.

$$
T_{\text {sum }}(t)=5+5 \cos (t)
$$

In the experiment of the proposed method, $\alpha, \beta$, and $\gamma$ were estimated prior to the experiment of the slip ratio difference control. The slip ratio difference control was conducted after completing the estimation because sufficient data for parameter estimation was not obtained in a single experiment. During parameters estimation, the torque distribution ratio was determined by using (63).

$$
K(t)=0.5+0.5 \cos (2 t)
$$

A slip ratio difference in the proposed method and the conventional method are shown in Fig. 10. Steady-state errors were found in the results obtained in the conventional method. These errors could be attributed to the lamp disturbances, which cannot be suppressed by disturbance observers. The difference of the slip ratio in the proposed method was obviously smaller than that in the conventional method as velocity-dependent parameters such as $\mu_{f}$ and $\mu_{r}$ were implicitly estimated.

The relation between the total torque and the torque distribution ratio of the proposed method is shown in Fig. 11. Note that the phase of $K$ was determined by $\beta$ and the bias of $K$ was determined by $\alpha$ and $\gamma$. As we see in Fig. 11, the phase of $K$ was different from that in the simulation, indicating estimation errors of $\beta$. Because the total torque was very small in the experiment, the reaction force hardly changed, making difficult the estimation of $\beta$, which is dependent on $T_{\text {sum }}$.

However, even though ideal $K$ was not determined, Fig. 10 shows the usefulness of the proposed method. Figure 12 shows an additional experimental result to help comprehension. In this experiment, $K$ linearly changed, and its relation 


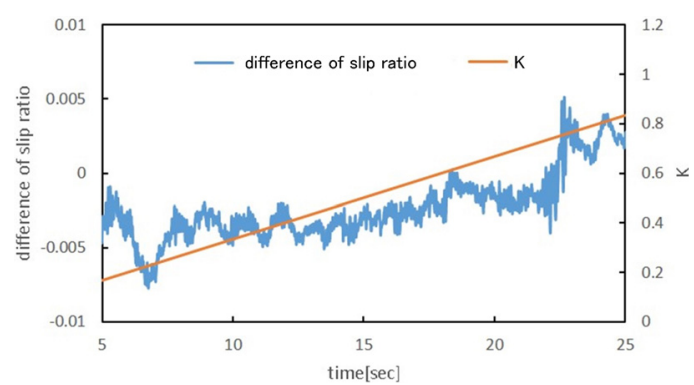

Fig. 13. Relation between torque distribution and slip ratio difference

with the slip ratio difference was studied. Figure 12 shows the dependency of the slip ratio difference on the torque distribution ratio. Figure 12 also shows if a wrong torque distribution ratio was used, the slip ratio differed. This fact indicates that $K$ was almost correctly estimated because of the small slip ratio difference, shown in Fig. 10. In other words, the bias of $K$ was correct and the variation of $K$ had few effects on the slip ratio difference because the variation of total torque was small.

\section{Conclusion}

In this paper, a torque distribution ratio control method for achieving the same slip ratio for both the front and rear wheel without knowing the reaction force distribution and vehicle's acceleration is proposed. In the proposed method, a model that represents the relation between the difference of each wheel's slip ratio and the torque distribution ratio was obtained from motion equations of two-wheeled vehicles. Then, the parameters were identified by the Kalman filter. As a result, the torque distribution ratio was appropriately adjusted by the proposed method, and the validity was verified in the simulation and the experiment. The proposed method can be widely used, because the proposed method can also be applied to four-wheeled electric vehicles.

\section{References}

( 1 ) A.V. Beznos, A.M. Formal'sky, E.V. Gurfinkel, and L.S. Tchesalin: "Control of autonomous motion of two-wheel bicycle with gyroscopic stablisation", in proceedings of IEEE International Conference on Robotics and Automation, pp.2670-2675 (1998)

( 2 ) M. Yamakita and A. Utano: "Automatic control of bicycle with a balancer", in Proceedings of IEEE/ASME International Conference on Advanced Intelligence Mechatronics, pp.1245-1249 (2005)

( 3 ) H. Niki and T. Murakami: "An approach to self stabilization of bicycle motion by handle controller", The Transactions of the Institute of Electrical Engineers of Japan D, A publication of Industry Applications Society, Vol.125, No.8, pp.779-785 (2005)

( 4 ) R.S. Sharp: "The stability and control of motorcycles", Journal of Mechanical Engineering Science, Vol.13, No.5, pp.316-329 (1971)

( 5 ) D. Soudbakhsh, Y. Zhang, and J. Yi: "Stability analysis of human balance control of stationary bicycles", in Proceedings of American Control Conference, pp.2755-2760 (2012)

( 6 ) T. Kawamura and H. Fujimoto: "Robust stabilization control for an electric bicycle", The Transactions of the Institute of Electrical Engineers of Japan, D, Vol.132, No.6, pp.651-658 (2012)

( 7 ) C. Yang and T. Murakami: "Full speed range self-balancing electric motorcycles without the handlebar", IEEE Transactions on Industrial Electronics, Vol.63, No.3, pp.1-12 (2016)

( 8 ) F. Chikhi, E. Hadri, and J. Cadiou: "Optimal control for anti-braking system", in Proceedings of IEEE International Symposium on Intelligent Control, pp.581-585 (2005)
(9) T.L. Lam, J. Yan, H. Qian, and Y. Xu: "Traction/braking force distribution algorithm for omni-directional all-wheel-independent-drive vehicles", in Proceedings of IEEE International Conference on Robotics and Automation Robotics and Automation, pp.746-751 (2013)

(10) Y. Syuiti and S. Takayuki: "Optimization of driving force distribution control in all-wheel drive based on wheel rotation speed difference between front and rear", Honda RED technical review 26, pp.110-115 (2014) (in Japanese)

(11) H. Fujimoto and S. Harada: "Model-based range extension control system for electric vehicles with front and rear driving-braking force distributions", IEEE Transactions on Industrial Electronics, Vol.62, No.5, pp.3245-3254 (2015)

(12) K. Fujii and H. Fujimoto: "Traction control based on slip ratio estimation for electric vehicle", in Proceedings of The Fourth Power Conversion Conference, Nagoya, pp.688-693 (2007)

(13) Y. Shi, K. Sun, L. Huang, and Y. Li: "Online identification of permanent magnet flux based on extended kalman filter for IMPSM drive with position sensorless control", IEEE Transacsions on Industrial Electronics, Vol.59, pp.4169-4178 (2012)

(14) P. Manganiello, M. Ricco, G. Petrone, E. Monmasson, and G. Spagunolo: "Dual-kalman-filter-based identification and real-time optimization of PV systems", IEEE Transacsions on Industrial Informatics, Vol.62, pp.41694178 (2015)

(15) S. Hong, C. Lee, F. Borelli, and J.K. Hedric: "A novel approach for vehicle inertial parameter identification using a dual kalman filter", IEEE Transacsions on Transportation Systems, Vol.16, pp.151-161 (2015)

(16) H. Kawajiri, H. Mizoguti, S. Sakaino, and T. Tsuji: "Sensorless pedaling torque estimation by front and rear wheels independently driven power assist bicycle", in Proceedings of IEEE International Conference on Mechatronics, pp.364-369 (2015)

(17) S. Sakaino, T. Sato, and K. Ohnishi: "Multi-dof micro macro bilateral controller using oblique coordinate control", IEEE Transacsions on Industrial Informatics, Vol.7, No.3, pp.446-454 (2011)

(18) K. Ohnishi, M. Shibata, and T. Murakami: "Motion control for advanced mechatronics", IEEE/ASME Transaction on Mechatronics, Vol.1, No.1, pp.56-67 (1996)

(19) H.B. Pacejka and E. Bakker: "The magic formula tyre model", in Proceedings of 1st International Colloquium on Tyre Models for Vehicle Dynamics Analysis, held in Delft (1991)

\section{Appendix}

Equation (22) can be transformed as follows:

$$
\begin{aligned}
& \frac{K T_{\text {sum }}}{A-B T_{\text {sum }}}-\frac{(1-K) T_{\text {sum }}}{C+D T_{\text {sum }}} \\
& \quad=\frac{K T_{\text {sum }}}{A}\left(1-\frac{B T_{\text {sum }}}{A}\right)^{-1}-\frac{(1-K) T_{\text {sum }}}{C}\left(1+\frac{D T_{\text {sum }}}{C}\right)^{-1} .
\end{aligned}
$$

Usually, $\left|\frac{B T_{\text {sum }}}{A}\right| \ll 1$ and $\left|\frac{D T_{\text {sum }}}{C}\right| \ll 1$ can be assumed. Then, Eq. (A1) is approximated as follows:

$$
\begin{aligned}
& \frac{K T_{\text {sum }}}{A}\left(1+\frac{B T_{\text {sum }}}{A}\right)-\frac{(1-K) T_{\text {sum }}}{C}\left(1-\frac{D T_{\text {sum }}}{C}\right) \\
& =-\frac{T_{\text {sum }}}{C}+\left(\frac{1}{A}+\frac{1}{C}\right) K T_{\text {sum }}+\frac{D}{C^{2}} T_{\text {sum }}^{2} \\
& \quad+\left(\frac{B}{A^{2}}-\frac{D}{C^{2}}\right) K T_{\text {sum }}^{2} \ldots \ldots \ldots \ldots \ldots \ldots \ldots \ldots
\end{aligned}
$$

Because $\frac{B}{A^{2}}$ and $\frac{D}{C^{2}}$ are approximately equal, the fourth term is neglected and finally the following linearized equation can be represented by three terms. The linearized equation is shown in (27).

$$
\begin{aligned}
& \lambda_{f}-\lambda_{r} \simeq T_{\text {sum }}\left(\alpha K+\beta T_{\text {sum }}+\gamma\right) \\
& \alpha=\frac{1}{A}+\frac{1}{C}, \quad \beta=\frac{D}{C^{2}}, \quad \gamma=-\frac{1}{C} .
\end{aligned}
$$


Sho Sakaino (Senior Member) received the B.E. degree in system

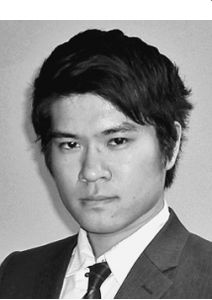
design engineering and the M.E. and Ph.D. degrees in integrated design engineering from Keio University, Yokohama, Japan, in 2006, 2008, and 2011, respectively. He is currently an assistant professor with Department of Electrical and Electronic Systems, Saitama University, Saitama, Japan. His research interests include mechatronics, motion control, robotics, and haptics. He received the Institute of Electrical Engineers of Japan Industry Application Society Distinguised Transaction Paper Award.

Hiroyuki Kawajiri (Non-member) received the B.E. and the M.E.

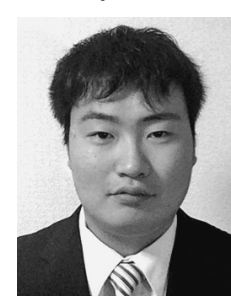
degrees in electrical and electronic system engineering from Saitama University, Saitama, Japan, in 2014 and 2016, respectively. He joined the NF CORPORATION in 2016. He currently works at TOKYO ELECTRON TOHOKU.
Toshiaki Tsuji (Senior Member) received the B.E., degree in system

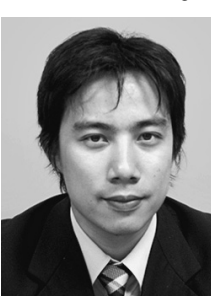
design engineering and the M.E. and Ph.D. degrees in integrated design engineering from Keio University, Yokohama, Japan, in 2006, 2008, and 2011, respectively. He was a research associate in Department of Mechanical Engineering, Tokyo University of Science from 2006 to 2007. He is currently with the Department of Electrical and Electronic Systems, Saitama University. His research interests include motion control, haptics, and rehabilitation robot. He received the FANUC FA and Robot Foundation Original Paper Award in 2007 and 2008 